\title{
The Parametric Degree of a Rational Surface
}

\author{
Josef Schicho* \\ Johann Radon Institute \\ Austrian Academy of Sciences
}

July 27, 2021

\begin{abstract}
The parametric degree of a rational surface is the degree of the polynomials in the smallest possible proper parametrization. An example shows that the parametric degree is not a geometric but an arithmetic concept, in the sense that it depends on the choice of the ground field. In this paper, we introduce two geometrical invariants of a rational surface, namely level and keel. These two numbers govern the parametric degree in the sense that there exist linear upper and lower bounds.
\end{abstract}

\section{Introduction}

The parametric degree of a rational surface is the degree of the polynomials in the smallest possible proper parametrization. In the absence of base points, the parametric degree is just the square root of the degree of the surface. On the other hand, there are examples of series of rational surfaces showing that the number of base points in the smallest parametrization can be much larger than the degree (see Example 6). It is therefore clear that the degree does not tell too much about the parametric degree of a rational surface.

Example 1below shows that the parametric degree is not a geometric but an arithmetic concept, in the sense that it depends on the choice of the ground field. For the complex case, [13] gave upper and lower estimations for the parametric degree in terms of the degree of the surface.

In this paper, we introduce two geometrical invariants of a rational surface, namely level and keel. These two numbers govern the parametric degree in the sense that there exist linear upper and lower bounds. In particular, level and keel determine the parametric degree up to a multiplicative factor of 2 , independent on the choice of the ground field (as long as it is perfect). We can therefore say that the parametric degree depends "just slightly" on the choice of the ground field, as this choice can change by a factor of at most 2. Of course,

${ }^{*}$ The author was supported by the FWF in the frame of the reseach projects SFB 1303 and P15551 
this is a bit misleading because there are surfaces for which rationality depends on the choice of the field, for instance surfaces that are rational over $\mathbb{C}$ but not rational over $\mathbb{R}$.

We note that this result implies that the question of rationality is decidable over any perfect field with decidable first order theory: first, we compute a parametrization over the algebraic closure, using [12. If it is of degree $d$, then, by the result in this paper, the surface is rational iff there is a parametrization of degree at most $2 d$. For a fixed surface, this question can be formulated as a first order sentence. Of course, decidability of rationality of surfaces is well-known for the real case by Comesatti's theorem (see [2, 14]).

\section{Parametric Degree}

Throughout the paper, we fix field $\mathbb{K}$, which is assumed to be perfect. A proper parametrization of a rational surface is a birational map $\nu: \mathbb{P}^{2} \rightarrow S \subset \mathbb{P}^{r}, r \geq 2$, which is defined over $\mathbb{K}$. We can write $\nu$ as

$$
\left(x_{0}: x_{1}: x_{2}\right) \mapsto\left(F_{0}\left(x_{0}, x_{1}, x_{2}\right) \ldots \ldots: F_{r}\left(x_{0}, x_{1}, x_{2}\right)\right),
$$

with $F_{0}, \ldots, F_{r} \in \mathbb{K}\left[x_{0}, x_{1}, x_{2}\right]$ homogeneous of the same degree $d$ and without a common divisor. This representation is unique up to the multiplication by a nonzero constant. The number $d$ is uniquely defined and it is called the degree of the parametrization. (This should not be mixed up with the concept of degree of a rational map; in fact, any proper parametrization is a rational map of degree 1.)

The surface $S$ has, in general, proper parametrizations of different degree. The smallest possible degree is called the parametric degree of $S$, and denoted by $\operatorname{pdeg}(S)$. For instance, we have $\operatorname{pdeg}\left(\mathbb{P}^{2}\right)=1$, and $\operatorname{pdeg}(Q)=2$ when $Q$ is a quadric surface in $\mathbb{P}^{2}$ with a $\mathbb{K}$-rational nonsingular point. In the second case, the inverse of the stereographic projection from this $\mathbb{K}$-rational point defines a parametrization of degree 2 .

The parametric degree is an arithmetic concept, i.e. it depends on the choice of the field $\mathbb{K}$.

Example 1. The torus with equation

$$
\left(x^{2}+y^{2}+z^{2}+\frac{16}{25} w^{2}\right)^{2}-4 x^{2} w^{2}-4 y^{2} w^{2}=0
$$

has a complex parametrization of degree 3, namely

$$
\begin{aligned}
(s: t: u) & \mapsto\left(\left(s^{2}+u^{2}\right)(3 u-8 t): i\left(s^{2}-u^{2}\right)(3 u-8 t):\right. \\
& -6 i s\left(u^{2}-6 t u+8 t^{2}: 20 s t(u-3 t)\right) .
\end{aligned}
$$

The smallest real parametrization has degree 4 . So we have $\operatorname{pdeg}\left(S_{\mathbb{C}}\right)=3$ and $\operatorname{pdeg}\left(S_{\mathbb{R}}\right)=4$. 


\section{Preliminaries}

Most definitions and theorems in this section are well-known. The single exception is the definition of the function nmc at the end of the section, which turns out to be convenient for the definition of the keel.

Let $S$ be a nonsingular projective surface over $\mathbb{K}$.

- A prime divisor is an irreducible curve on $S$. Note that it is required that the curve is defined over $\mathbb{K}$, but it may split into several components over the algebraic closure.

- $\operatorname{Div}(S)$, the group of divisors of $S$, is the free abelian group generated by the prime divisor. Divisors are denoted by captital letters.

- A divisor is called effective iff all its coefficients are nonnegative. If $B-A$ is effective, then we also say that $A$ divides $B$ or $A \leq B$.

- Two effective divisor without common component are equivalent iff they are two fibers of a rational map $S \rightarrow \mathbb{P}^{1}$. Linear equivalence is the finest equivalence relation on $\operatorname{Div}(S)$ which is compatible with addition and for which the previous statement is true.

- $\operatorname{Pic}(S)$ is the group of classes of divisors. Classes are denoted by capital letters.

- The class $D$ is called effective iff it has an effective divisor.

- $|D|$ is the set of all effective divisors in the class $D$. It has a natural structure of a projective space over $\mathbb{K}$. The dimension of this projective space is denoted by $\operatorname{dim}(D)$. A linear system of divisors is a subset of $|D|$ corresponding to a projective subspace.

- If the point $p \in S$ is contained in all divisors in a linear system $l$, then $p$ is a base point of $l$. If the complete linear system $|D|$ has no base points, then we say that $D$ is free.

- If $l$ is a non-empty linear system, then the associated rational map is denoted by $\phi_{l}: S \rightarrow \mathbb{P}^{\operatorname{dim}(l)}$. It is defined outside the base locus. The codomain $\mathbb{P}^{\operatorname{dim}(l)}$ is naturally identified with the dual projective space of $l$; the image of $p$ corresponds to the subset of divisors in $l$ passing through $p$, which is a hyperplane.

- The intersection product $\operatorname{Pic}(S)^{2} \rightarrow \mathbb{Z}$ is symmetric and bi-additive, and if the classes $A$ resp. $B$ contain two effective divisors $A_{0}$ resps. $B_{0}$ without common component, then $A B$ is the number of common points of $A_{0}$ and $B_{0}$, properly counted. Especially, $A B \geq 0$ in this case.

- $K$ or $K_{S}$ is the canonical class of $S$.

- A class $D$ is called nef iff $D C \geq 0$ for all effective $C$. 
We also need the following well-known theorems.

Theorem 1. A birational regular map $\phi: S_{1} \rightarrow S_{2}$ induces two homomorphisms, the pushforward $\phi_{*}: \operatorname{Div}\left(S_{1}\right) \rightarrow \operatorname{Div}\left(S_{2}\right)$ and the pullback $\phi^{*}: \operatorname{Div}\left(S_{2}\right) \rightarrow$ $\operatorname{Div}\left(S_{1}\right)$. Both functions are well defined on classes and preserve effectivity.

For $C \in \operatorname{Pic}\left(S_{1}\right)$ and $D \in \operatorname{Pic}\left(S_{2}\right)$, the following hold.

- $\left(\phi_{*} \circ \phi^{*}\right)(D)=D$.

- $\operatorname{dim}\left(\phi_{*}(C)\right) \geq \operatorname{dim}(C)$.

- $\operatorname{dim}\left(\phi^{*}(D)\right)=\operatorname{dim}(D)$.

- $\phi_{*}(C) D=C \phi^{*}(D)$.

- $\left(\phi_{*}(C)\right)^{2} \geq C^{2}$.

- $\left(\phi^{*}(D)\right)^{2}=D^{2}$.

- $\phi_{*}\left(K_{S_{1}}\right)=K_{S_{2}}$.

Theorem 2. Let $S$ be a nonsingular projective surface. Let $E \in \operatorname{Div}(S)$ be a prime divisor such that $E^{2}=E K<0$. A prime divisor with these properties is called exceptional divisor.

Then there exists a regular birational map $\pi: S \rightarrow S^{\prime}$, called the blowing down of $E$, such that the kernel of $\pi_{*}$ is generated by $E$. Moreover, $K_{S}=$ $\pi^{*}\left(K_{S^{\prime}}\right)+E$.

Any birational regular map is a composition of such blowing down maps.

We define a function nmc (for "number of moving components") from effective classes to nonnegative integers. Let $X(D) \subset \mathbb{P}^{\operatorname{dim}(D)}$ be the image of the associated rational map $\phi_{D}$.

- If $X(D)$ is a point (this is the case iff $\operatorname{dim}(D)=0$ ), then $\operatorname{nmc}(D):=0$.

- If $X(D)$ is a curve of degree $m$, then $\operatorname{nmc}(D):=m$.

- If $X(D)$ is a surface, then $\operatorname{nmc}(D):=1$.

\section{$3 \quad$ Level and Keel}

We first introduce level and keel for divisors on nonsingular surfaces. Then the concepts are transferred to embedded surfaces with arbitrary singularities, using a resolution of singularities.

Let $D$ be an effective divisor class of $S$. The level of $D$ is the supremum of all rational numbers $p / q, q>0$, such that $q D+p K$ is effective. If the supremum is assumed, then the keel of $D$ is equal to the supremum of all numbers of the form $\frac{\operatorname{nmc}(q D+p K)}{q}$ where $p / q$ is the level. If the supremum in the definition of the level is not assumed (for instance if the level is irrational or infinity), then the keel is defined as 0 . 
Remark 1. If some multiple of $K$ is effective, then we have level $(D)=\infty$ and $\operatorname{keel}(D)=0$. Hence level and keel are only interesting if the Kodaira dimension of $S$ is negative.

Remark 2. The numbers $\operatorname{dim}(D)$ and $\operatorname{nmc}(D)$ are preserved under extension of the ground field. It follows that level and keel are preserved under extension of the ground field (i.e. they are geometric).

Example 2. Let $S=\mathbb{P}^{2}$ and $D=n L$, where $L$ is the class of lines and $n \geq 0$. Then $K=-3 L$, and a class $m L$ is effective iff $m \geq 0$, and we have nmc $(0)=0$. It follows that $\operatorname{level}(D)=n / 3$ and $\operatorname{keel}(D)=0$.

Example 3. Let $S=\mathbb{P}^{1} \times \mathbb{P}^{1}$ and $D=m F_{1}+n F_{2}$, where $F_{1}, F_{2}$ are the classes of the fibers of the two projections and $0 \leq m \leq n$. Then $K=-2 F_{1}-2 F_{2}$, and a class $a F_{1}+b F_{2}$ is effective iff $a \geq 0$ and $b \geq 0$, and we have $\operatorname{nmc}\left(a F_{2}\right)=a$. It follows that $\operatorname{level}(D)=m / 2$ and $\operatorname{keel}(D)=n-m$.

Example 4. We sketch an example which generalizes the two above.
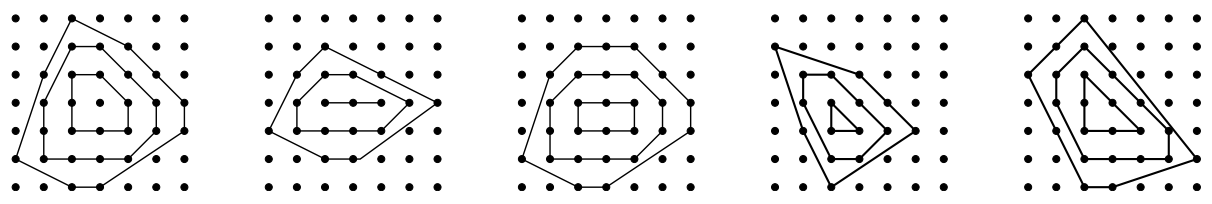

Figure 1: Polygons of level and keel $(3,0),(2,2),(5 / 2,1),(7 / 3,0)$, and $(8 / 3,0)$.

Let $\Gamma$ be a convex lattice polygon, i.e. the convex hull of a finite number of points in the plane with integer coordinates. The polygon $\Gamma$ defines a nonsingular toric surface $S$ (the minimal resolution of the toric surface defined by the inner normals) and an effective divisor (the inverse image of the class of hyperplane section in the projective embedding defined by $\Gamma$ ).

The class $q D+p K$ corresponds to the convex figure obtained by scaling $\Gamma$ by a factor of $q$ and moving each edge $p$ steps inward. The class is effective iff this figure is non-empty. Hence the level is equal to $p / q$ if we can enlarge $\Gamma$ by a factor of $q$, pass $p$ times to the convex hull of the interior points, and obtain a line segment or a point (see figure 1 cf also [3]). The keel is the number of points on this line segment or point, minus 1 , divided by $q$.

We now define level and keel of a projective surface $S \subset \mathbb{P}^{r}$ (possibly with singularities). Let $\pi: \tilde{S} \rightarrow S$ be a resolution of singularities, i.e. a proper birational map such that $\tilde{S}$ is nonsingular. Let $H \in \operatorname{Pic}(\tilde{S})$ be the class of the pullbacks of hyperplane sections. We define the level and the keel of $S$ as the level and the keel of $H$. Theorem 4 below says that this is independent of the choice of the desingularization.

Lemma 3. Let $\phi: S_{1} \rightarrow S_{2}$ be a regular birational map. Let $D \in \operatorname{Pic}\left(S_{2}\right)$. Let $C$ be an effective divisor of $S_{1}$ such that $\phi_{*}(C)=0$. Then $C$ is a common divisor of the linear system $\left|\phi^{*}(D)+C\right|$. 
Proof. The proof proceeds by induction on the number of blowing downs into which the birational regular map $\phi$ can be decomposed. First, assume that $\phi: S_{1} \rightarrow S_{2}$ is the blowing down of an exceptional divisor $E$. Then $C=n E$ for some $n \geq 0$. Let $m \geq 0$ be the largest number such that $m E$ is a common divisor of $\left|\phi^{*}(D)+n E\right|$. Then there exists an effective divisor in $\left|\phi^{*}(D)+(n-m) E\right|$ that does not have $E$ as component. It follows that $\left(\phi^{*}(D)+(n-m) E\right) E=m-n \geq 0$, which shows that $n E$ is a common divisor.

Second, assume that $\phi$ can be decomposed as $S_{1} \stackrel{\phi_{1}}{\rightarrow} S_{3} \stackrel{\phi_{2}}{\rightarrow} S_{2}$, where $\phi_{1}$ is the blowing down of an exceptional divisor $E$. Because the pushforward preserves effectivity, $\phi_{1_{*}}(C)$ is effective. By induction, $\phi_{1_{*}}(C)$ is a common divisor of the linear system $\left|\phi_{2}{ }^{*}(D)+\phi_{1 *}(C)\right|$. Because the dimension of a linear system is preserved by pullback, the equation $\operatorname{dim}\left(\phi_{2}{ }^{*}(D)+\phi_{1 *}(C)\right)=\operatorname{dim}\left(\phi_{2}{ }^{*}(D)\right)$ implies the equation $\operatorname{dim}\left(\phi^{*}(D)+\phi_{1}{ }^{*} \phi_{1_{*}}(C)\right)=\operatorname{dim}\left(\phi^{*}(D)\right)$, which implies that $\phi_{1}{ }^{*} \phi_{1 *}(C)$ is a common divisor of $\left|\phi^{*}(D)+\phi_{1}{ }^{*} \phi_{1 *}(C)\right|$. Now $C-\phi_{1}{ }^{*} \phi_{1 *}(C)$ lies in the kernel of $\phi_{1_{*}}$. Therefore it is a multiple $n E$ of the exceptional divisor.

We distinguish two cases. If $n \leq 0$, then $C \leq \phi_{1}{ }^{*} \phi_{1 *}(C)$, and it follows

$$
\operatorname{dim}\left(\phi^{*}(D)\right) \leq \operatorname{dim}\left(\phi^{*}(D)+C\right) \leq \operatorname{dim}\left(\phi^{*}(D)+\phi_{1}{ }^{*} \phi_{1 *}(C)\right)=\operatorname{dim}\left(\phi^{*}(D)\right),
$$

hence we have equality everywhere and the statement is proved.

If $n>0$, then $n E$ is effective, and $n E$ is a common divisor of the linear system $\left|\phi_{1}{ }^{*}\left(\phi_{2}{ }^{*}(D)+\phi_{1 *}(C)\right)+n E\right|$ by the induction base case. Therefore, we have

$$
\begin{gathered}
\operatorname{dim}\left(\phi^{*}(D)+C\right)=\operatorname{dim}\left(\phi_{1}{ }^{*}\left(\phi_{2}{ }^{*}(D)+\phi_{1 *}(C)\right)+n E\right)= \\
\operatorname{dim}\left(\phi_{1}{ }^{*}\left(\phi_{2}{ }^{*}(D)+\phi_{1 *}(C)\right)\right)=\operatorname{dim}\left(\phi^{*}(D)\right),
\end{gathered}
$$

hence the statement is also proved.

Theorem 4. Let $\pi_{1}: \tilde{S}_{1} \rightarrow S$ and $\pi_{2}: \tilde{S}_{2} \rightarrow S$ be two desingularizations of S. Let $H_{1} \in \operatorname{Pic}\left(\tilde{S}_{1}\right)$ and $H_{2} \in \operatorname{Pic}\left(\tilde{S}_{2}\right)$ be the pullbacks of hyperplane sections. Then we have

$$
\operatorname{level}\left(H_{1}\right)=\operatorname{level}\left(H_{2}\right), \operatorname{keel}\left(H_{1}\right)=\operatorname{keel}\left(H_{2}\right) .
$$

Proof. First, let us assume that there exists a birational regular map $\phi: \tilde{S}_{1} \rightarrow \tilde{S}_{2}$ such that $\pi_{1}=\pi_{2} \circ \phi$. Then $\phi$ transforms hyperplane pullbacks to hyperplane pullbacks, i.e. $\phi^{*}\left(H_{1}\right)=H_{2}$. Let $p, q$ be positive integers. By Theorem 2 the class $C:=K_{1}-\phi^{*}\left(K_{2}\right)$ is effective. By Lemma 3 the divisor $p C$ is a common divisor of the linear system $\left|\phi^{*}\left(q H_{2}+p K_{2}\right)+p C\right|=\left|q H_{1}+p K_{1}\right|$. It follows that the two linear systems $\left|q H_{2}+p K_{2}\right|$ and $\left|q H_{1}+p K_{1}\right|$ have the same dimension and the same number of moving components, and the statement is proven.

In the general case, there exists a dominating desingularization $\pi_{3}: \tilde{S}_{3} \rightarrow S$ and birational regular maps $\phi_{i}: \tilde{S}_{3} \rightarrow \tilde{S}_{i}$ such that $\pi_{3}=\pi_{i} \circ \phi_{i}$ for $i=1,2$. Hence it can be reduced to the special case above.

Example 5. Assume that $\nu: \mathbb{P}^{2} \rightarrow S$ is a parametrization of degree $d$ without base points. Then $\nu$ is regular and we can use it as resolution of singularities. It follows that $H=d L$ and we have $\operatorname{level}(S)=\operatorname{level}(H)=d / 3$ and $\operatorname{keel}(S)=$ $\operatorname{keel}(H)=0$, by Example 2 
It is also convenient to extend the notion of parametric degree to divisors. Let $S$ be a nonsingular surface. Let $D \in \operatorname{Pic}(S)$ be an nef divisor. A linear system $l$ of divisors is called parametrizing iff $\operatorname{dim}(l)=2$ and $\phi_{l}: S \rightarrow \mathbb{P}^{2}$ is birational. A class $P$ is called parametrizing iff $|P|$ contains a parametrizing linear system. Then we define $\operatorname{pdeg}(D)$ as the minimum of all numbers $P D$, where $P$ is a parametrizing class.

Lemma 5. Let $S \subset \mathbb{P}^{r}$ be a (possibly singular) surface. Let $\pi: \tilde{S} \rightarrow S$ be a resolution of its singularities. Let $H \in \operatorname{Pic}(\tilde{S})$ be the class of the pullbacks of hyperplane sections. Then $\operatorname{pdeg}(S)=\operatorname{pdeg}(H)$.

Proof. There is a one-to-one correspondence of parametrizations of $S$ and parametrizing linear systems of $D$, and the degrees coincide for corresponding parametrization/class.

Remark 3. Lemma 5 allows to reduce any relation between parametric degree, level and keel of a singular surfaces to the same relation between parametric degree, level and keel of a divisor on a nonsingular surface.

\section{The Lower Bound}

The main idea for establishing a lower bound for the parametric degree in terms of level and keel is to analyze what happens in the examples 2 and 5 when the parametrization has base points.

Theorem 6. Let $S \subset \mathbb{P}^{r}$ be a rational surface. Then we have

$$
\operatorname{pdeg}(S) \geq 3 \operatorname{level}(S)+\operatorname{keel}(S) .
$$

Proof. It suffices to prove the above inequality for a divisor $H$. We assume that $P H=\operatorname{pdeg}(H)$ and that some linear subsystem of $|P|$ induces a birational map to $\mathbb{P}^{2}$. Moreover, we assume that $l \subseteq|P|$ is a parametrizing linear system. If $C$ is a common divisor of $l$, then we would have $(P-C) H \leq P H$ because $H$ is nef. In this case, we can replace $P$ by $P-C$ which is also a parametrizing class. As we can do this only finitely many times, because $l$ has only finitely many common components, we can assume that $l$ has no common components.

Claim 1: $P$ is nef. Indeed, if $C$ is a prime divisor, then $C P \geq 0$ because $C$ is not a common component of $l$.

Claim 2: if $C$ is a prime divisor with positive dimension, then $P C>0$. Indeed, we have $P^{2}>0$ because the image of $\phi_{l}$ is $\mathbb{P}^{2}$, and therefore two generic divisors in $l$ intersect in a point outside the base locus. And $|C|$ contains two divisors without common component, hence $C^{2} \geq 0$. Therefore $P C$ cannot be zero by the Hodge index theorem.

Claim 3: $P K \leq-3$. To prove this, we resolve the base points of $\phi_{l}: S \rightarrow \mathbb{P}^{2}$ and get a birational regular map $\pi: \tilde{S} \rightarrow S$ such that $\phi_{P} \circ \pi: \tilde{S} \rightarrow \mathbb{P}^{2}$ is regular. Then $\pi^{*}(P)$ is nef by Theorem 2 and it follows

$$
P K=\pi^{*}(P) \pi^{*}(K)=\pi^{*}(P) K_{\tilde{S}} \leq \pi^{*}(P)\left(\phi_{l} \circ \pi\right)^{*}\left(K_{\mathbb{P}^{2}}\right)
$$




$$
=\left(\phi_{l} \circ \pi\right)_{*}\left(\pi^{*}(P)\right) K_{\mathbb{P}^{2}}=L(-3 L)=-3,
$$

where $L \in \operatorname{Pic}\left(\mathbb{P}^{2}\right)$ is the class of lines.

For two positive integers $p, q$, the divisor $q H+p K$ can be effective only if $(q H+p K) P=q \operatorname{pdeg}(H)-3 p \geq 0$. This proves that level $(H) \leq \operatorname{pdeg}(H) / 3$.

Now, assume $p / q=\operatorname{level}(H)$. We claim that $\operatorname{nmc}(q H+p K) \leq \operatorname{pdeg}(H) q-$ $3 p$. Let $F$ be the greatest common divisor of $q H+p K$, and let $B$ be a generic divisor in $|q H+p K-F|$. Then $B$ corresponds to a generic hyperplane section of the associated image $X:=\phi_{q H+p K}(S)$. If the image $X$ is a point, then $\operatorname{nmc}(q H+p K)=0$ and the claim is true. If $X$ is a surface, then $B$ has positive dimension; it follows that $\operatorname{pdeg}(H) q-3 p \geq B P \geq 1$, and because $\operatorname{nmc}(q H+p K)=1$ the claim is true. If $X$ is a curve, then $X$ is necessarily rational, and $\phi_{q H+p K}$ factors through a rational map $S \rightarrow \mathbb{P}^{1}$ which is associated to some divisor $A$. Because the divisors in $A$ are also the fibers of $\phi_{q H+p K}$, we have $B=m A$, where $m=\operatorname{nmc}(q H+p K)$ is the number of intersection points of $X$ with a generic hyperplane. Since $A$ has positive dimension, we have

$$
\operatorname{pdeg}(H) q-3 p \geq B P=m A P \geq m=\operatorname{nmc}(q H+p K),
$$

hence the claim is true also in this last case. This shows that

$$
\operatorname{keel}(H) \leq \operatorname{pdeg}(H)-3 p / q=\operatorname{pdeg}(H)-\operatorname{level}(H) .
$$

Remark 4. By analyzing Example 3 in a similar fashion, one can show that if $S$ has a parametrization of bidegree $(m, n), m \leq n$, then $m \geq 2 \operatorname{level}(S)$ and $n \geq 2 \operatorname{level}(S)+\operatorname{keel}(S)$.

Example 6. Here is an example that shows how to use the concepts of level and keel in order to construct surfaces that have a high parametric degree.

Let $n \geq 5$ be an odd integer. Let $S \subset \mathbb{P}^{3}$ be the surface given by the equation

$$
z^{2 n+1}-x^{2} w^{2 n-1}-y^{n} w^{n+1}=0 .
$$

We can compute the level and keel by using the parametrization

$$
\begin{aligned}
(s: t: u) \mapsto & \left(\left(s^{2} u^{n-2}+t^{n}\right)^{n} s:\left(s^{2} u^{n-2}+t^{n}\right)^{2} t u^{n^{2}-2 n}\right. \\
& \left.:\left(s^{2} u^{n-2}+t^{n}\right) u^{n^{2}-n+1}: u^{n^{2}+1}\right) .
\end{aligned}
$$

By resolving the base points of the parametrization, we get a resolution $\tilde{S}$ of the singularities of $S$. Explicit analysis of the base points (see [15]) shows that there is one base point of multiplicity $n^{2}-2 n$, with $\frac{n-3}{2}$ base points of multiplicity $2 n$ and $2 n+3$ base points of multiplicity $n$ in the infinitely near, and one simple base point with $n^{2}-2 n-1$ simple base points in the infinitely near.

For positive integers $p, q$, the linear system $|q H+p K|$ on $\tilde{S}$ corresponds to the linear space of forms of degree $q\left(n^{2}+1\right)-3 p$ that vanish with multiplicity $q r-p$ at each point of multiplicity $r$. Such forms exist for $2 p \leq(2 n+1) q$, hence 
level $(S)=n+\frac{1}{2}$. If $2 p=(2 n+1) q$, then the corresponding linear space is the vectorspace of forms of degree $\frac{2 n^{2}-6 n-1}{2} q$ vanishing with multiplicity $\frac{2 n^{2}-6 n-1}{2} q$ at the $\left(n^{2}-2 n\right)$-fold base point and with multiplicity $\frac{2 n-1}{2} q$ at the $\frac{n-3}{2}$ base points of multiplicity $2 n$ in the infinitely near. Hence

$$
\operatorname{keel}(S)=\frac{2 n^{2}-6 n-1}{2}-\frac{n-3}{2} \frac{2 n-1}{2}=\frac{2 n^{2}-5 n-5}{4} .
$$

By Theorem [6] the parametric degree is greater than or equal to $\frac{2 n^{2}+7 n+1}{4}$. Because there is a parametrization of degree $n^{2}+1$, we know that the parametric degree grows proportional to the square of the implicit degree.

\section{The Adjoint Chain}

Adjoints are a tool for constructing minimal models of a given surface or higherdimensional varieties. Starting with a nef divisor class, we keep alternating to blow down orthogonal exceptional divisors and adding the canonical class, until nefness does not hold any more. The last surface with nef class in the process is a minimal model with special properties. This technique has been used in 12 to construct parametrizations in the case $\mathbb{K}$ is algebraically closed. Similar constructions appear in various other contexts, see [1] for a survey.

Let $S$ be a nonsingular surface, and let $D \in \operatorname{Pic}(S)$. Following [9, 10, we say that $S$ is $D$-minimal iff $S$ has no exceptional divisor orthogonal to $D$. We say $S$ is minimal iff $S$ is 0 -minimal. The following theorem is well-known (see [9]).

Theorem 7. Let $S$ be a nonsingular surface and let $D \in \operatorname{Pic}(S)$. Then there exists a birational regular map $\mu: S \rightarrow S_{0}$, such that $D=\left(\mu^{*} \circ \mu_{*}\right)(D)$ and $S_{0}$ is $\mu_{*}(D)$-minimal. We call this a D-minimalization.

In the rest of the paper, we fix a nonsingular rational surface $S$ and a nef class $D \in \operatorname{Pic}(S)$ such that $D^{2}>0$ (for instance, the class of pullback of hyperplane sections in a resolution). The adjoint chain $\mathcal{S}$ is a chain of surfaces and birational regular maps

$$
S \stackrel{\mu_{0}}{\rightarrow} S_{0} \stackrel{\mu_{1}}{\rightarrow} S_{1} \stackrel{\mu_{2}}{\rightarrow} \ldots
$$

and divisor classes $D_{i} \in \operatorname{Pic}\left(S_{i}\right)$, which is constructed recursively in the following way. First, we let $\mu_{0}: S \rightarrow S_{0}$ be a $D$-minimalization of $S$, and we let $D_{0}:=$ $\mu_{0 *}\left(D_{0}\right)$. Now assume that we have already defined $S_{i}$ and $D_{i}$. Let $K_{i}$ be the canonical class of $S_{i}$. If $D_{i}+K_{i}$ is not effective, then the adjoint chain ends; we denote the index of the last surface with $a$. Otherwise, we let $\mu_{i+1}: S_{i} \rightarrow S_{i+1}$ be a $\left(D_{i}+K_{i}\right)$-minimalization of $S_{i}$, and we let $D_{i+1}:=\left(\mu_{i+1}\right)_{*}\left(D_{i}+K_{i}\right)$. If the adjoint chain is infinite, then we set $a:=\infty$ (but we will prove that $a$ is finite).

Lemma 8. The classes $D_{i}$ above are effective and nef. If $i<a$, then $D_{i}{ }^{2}>0$. 
Proof. If $\mathbb{K}$ is algebraically closed, then the proof is well-known ([12], Lemma A.2 and Lemma A.3). There is only one step in the proofs of [12] that uses the assumption that $\mathbb{K}$ is algebraically closed: in this case, any prime divisor $C$ of dimension 0 with $C K<0$ is exceptional, and we have $C^{2}=C K=-1$ (Lemma A.1 in [12]). It follows that if $D$ is nef and $D+K$ is effective but not nef, then there exists an exceptional divisor orthogonal to $D$.

Here is an adaption of the proof to the case of non-closed fields: assume that $D$ is nef and $D+K$ is effective but not nef. Let $C$ be a prime divisor such that $(D+K) C<0$. Then $C K<0$, and $\operatorname{dim}(C)=0$ because $C$ must be fixed in $|D+K|$. By the lemma below, $C$ is exceptional and $C D$ is an integral multiple of $C K$. This is only possible if $C D=0$, hence $C$ is orthogonal to $D$, and the rest of the proof works as in the case where $\mathbb{K}$ is algebraically closed.

Lemma 9. Let $C$ be a prime divisor such that $\operatorname{dim}(C)=0$ and $C K<0$. Then $C$ is exceptional, and $C D$ is an integral multiple of $C K$ for all $D \in \operatorname{Pic}(D)$.

Proof. Let $\bar{S}$ be the surface obtained by base field extension to the algebraic closure $\overline{\mathbb{K}}$. There are natural injections $\operatorname{Div}(S) \rightarrow \operatorname{Div}(\bar{S})$ and $\operatorname{Pic}(S) \rightarrow \operatorname{Pic}(\bar{S})$. In general, $C$ need not be a prime divisor in $\operatorname{Div}(\bar{S})$, but it has only simple components $C=\sum_{i=1}^{r} C_{i}$. Each $C_{i}$ has dimension 0 . Moreover, $C_{i} D=C_{j} D$ for any $i, j \leq r$ and $D$ coming from $S$, because $C_{i}$ and $C_{j}$ are conjugate under the action of the Galois group of the extension $\mathbb{K} \subset \overline{\mathbb{K}}$. Especially, $C_{i} K=C_{j} K$. It follows that $C_{i} K<0$. By Lemma A.1 in 12, each $C_{i}$ is exceptional, and $C_{i}^{2}=C_{i} K=-1$ for all $i$. Hence $C K=-r$, and $C D$ is an integral multiple of $r$.

It remains to show that $C^{2}=-r$. For $i \neq j$, we have $\operatorname{dim}\left(C_{i}+C_{j}\right) \geq C_{i} C_{j}$ by the Riemann-Roch theorem. On the other hand, $\operatorname{dim}\left(C_{i}+C_{j}\right)=0$ because $\operatorname{dim}(C)=0$, hence $C_{i} C_{j}=0$. Hence $C^{2}=\sum_{i=1}^{r} C_{i}^{2}=-r$.

Lemma 10. Let $p, q, i$ be integers such that $i \leq a$ and $p \geq q i$. Then the linear systems $|q D+p K|$ and $\left|q D_{i}+(p-q i) K_{i}\right|$ have the same dimension and number of moving components.

Proof. For $j=0, \ldots, i$, let $\phi_{j}: S \rightarrow S_{j}$ be the map $\mu_{j} \circ \cdots \circ \mu_{0}$. Then $\phi_{j}^{*}\left(D_{j}\right)=\phi_{j-1}^{*}\left(D_{j-1}+K_{j-1}\right)$. The class $E_{j}:=\phi_{j}^{*} K_{j}-\phi_{j-1}^{*} K_{j-1}$ is effective. Therefore, the class

$$
C:=q D+p K-\phi_{i}^{*}\left(q D_{i}+(p-q i) K_{i}\right)=\sum_{j=0}^{i}(p-q j) E_{j}
$$

is effective, too. By Lemma 3 the unique divisor in $|C|$ is fixed in $\mid q D+$ $p K \mid$. Because the pullback preserves the dimension and the number of moving components, the lemma follows.

Corollary 11. We have level $\left(D_{i}\right)=\operatorname{level}(D)-i$ and $\operatorname{keel}\left(D_{i}\right)=\operatorname{keel}(D)$.

Lemma 12. We have $a \leq \operatorname{level}(D)$. In particular, a is finite. 
Proof. By Corollary 11] it suffices to prove that level $\left(D_{a}\right) \geq 0$. But this is clear since $D_{a}$ is effective.

The following lemmas can be used to compute level and keel in terms of the adjoint chain.

Lemma 13. Assume that $D_{a}=0$. Then $\operatorname{level}(D)=a$ and $\operatorname{keel}(D)=0$.

Proof. This follows immediately from Corollary 11 and from level $(0)=0$ and $\operatorname{keel}(0)=0$.

Lemma 14. Assume that $D_{a}{ }^{2}=0$ and $D_{a} \neq 0$. Then $\operatorname{level}(D)=a$ and $\operatorname{keel}(D)=\operatorname{nmc}\left(D_{a}\right)=: k>0$, and there is a free divisor $P \in \operatorname{Pic}\left(S_{a}\right)$ such that $D_{a}=k P$ and $P K_{a}=-2$ and $P^{2}=0$ and $\operatorname{dim}(P)=1$.

Proof. The proof of Lemma A.7 in 12 generalizes without problems to nonclosed fields. This shows the existence of $P$ with the desired properties.

If $p, q>0$, then $q D_{a}+p K_{a}$ cannot be effective because $\left(q D_{a}+p K_{a}\right) P=$ $-2 p<0$. Hence $\operatorname{level}\left(D_{a}\right)=0$ and $\operatorname{level}(D)=a$ by Corollary 11 Moreover, $\operatorname{nmc}\left(q D_{a}\right)=\operatorname{nmc}(q k P)=q k, \operatorname{hence} \operatorname{keel}\left(D_{a}\right)=\operatorname{keel}(D)=k$.

Lemma 15. Assume that $D_{a}{ }^{2}>0$. Then one of the following cases holds.

a) $\operatorname{level}(D)=a+1 / 3, \operatorname{keel}(D)=0$, and $3 D_{a}+K_{a}=0$.

b) $\operatorname{level}(D)=a+2 / 3, \operatorname{keel}(D)=0$, and $3 D_{a}+2 K_{a}=0$.

c) $\operatorname{level}(D)=a+1 / 2, \operatorname{keel}(D)=0$, and $2 D_{a}+K_{a}=0$.

d) $\operatorname{level}(D)=a+1 / 2, \operatorname{keel}(D)=\operatorname{nmc}\left(2 D_{a}+K_{a}\right) / 2>0$, and $\left(2 D_{a}+K_{a}\right)^{2}=0$. In particular, level and keel are rational numbers with a denominator dividing 6 .

Proof. Lemma A.8 from 12 - which is also true in the case $\mathbb{K}$ is non-closed - says that we can conclude from $D_{a}{ }^{2}>0$ that either $3 D_{a}+K_{a}=0$, or $3 D_{a}+2 K_{a}=0$, or $\left(2 D_{a}+K_{a}\right)^{2}=0$. Using Corollary 11] we get level $(D)=\operatorname{level}\left(D_{a}\right)+a=a+i / 3$ in the $i$-th case, for $i=1,2$. In the third case, we apply Lemma 13 or Lemma 14 to $D:=2 D_{a}+K_{a}$, and we get either (c) or (d), depending whether $2 D_{a}+K_{a}$ is zero or not.

Remark 5. At this point, it is instructive to revisit Example 4 again. Starting from a convex lattice polygon, we pass to the convex hull of the interior points $a$ times. If we obtain a single point, then $D_{a}=0$ holds for the corresponding toric surface. If we obtain a line segment with $k+1$ lattice points, then $D_{a}=k P$ for some $P$ with $P^{2}=0$ and $P K_{a}=-2$, as in Lemma 14 If we obtain a lattice polygon without interior lattice points, then one of the following four cases holds:

a) after scaling by 3 , we get a polygon with one interior point;

b) after scaling by 3 and passing to the convex hull of interior points, we get a polygon with one interior point;

c) after scaling by 2 , we get a polygon with one interior point;

d) after scaling by 2 , we get a polygon with several interior points that are all on a line.

Of course, these are instances of the 4 cases (a), (b), (c), (d) in Lemma 15. 
Remark 6. The lemmas above remind on the Kawamata Rationality Theorem and the Kawamata-Shokurov Base Point Free Theorem (see [8]): if $D$ is ample, then the "nefness value" $v$ is a rational number, and some multiple of $D+v K$ is free. The associated contraction morphism is either a blowing down, or a map with conic fibers, or a constant map. Of course, the Kawamata Rationality Theorem and the Kawamata-Shokurov Base Point Free Theorem hold in a much more general context (arbitrary dimension, rationality need not be assumed).

Remark 7. If $S$ is a rational surface with degree $d$ and sectional genus $p_{1}$, then we have the inequality $a+\operatorname{dim}\left(D_{a}\right) \leq p_{1}+\left(\begin{array}{c}2 p_{1}-d-1 \\ 2\end{array}\right)$, by Lemma 8 in 13. Using the classification of surfaces occuring in Lemma 15 (see Lemma A.8 in [12]), it is easy to check that $\operatorname{keel}(D) \leq \operatorname{dim}\left(D_{a}\right)$ in all cases. Together with the upper bound $p_{1} \leq\left(\begin{array}{c}d-1 \\ 2\end{array}\right)$ for the sectional genus, we get the bound level $(D)+\operatorname{keel}(D) \leq d^{4} / 2$. Together with the bound in Theorem 20 below, we obtain the bound $\operatorname{pdeg}(S) \leq 3 \operatorname{deg}(S)^{4}$.

\section{The Upper Bound}

In order to establish an upper bound for the parametric degree, one has to construct a parametrization (or, equivalently, a parametrizing divisor class). The idea is to construct a minimal model using adjoints, and then to use the wellknown classification of such minimal surfaces, due to Manin and Iskovskih 9, 10, 4, 5, 6, 7.

Theorem 16. Let $S$ be a minimal rational surface such that $-K$ is nef and $K^{2}>0$. Then one of the following cases holds.

a) $S \cong \mathbb{P}^{2}$; in this case, $K=-3 L$, where $L$ is the class of lines.

b) $S$ is isomorphic to a quadric in $\mathbb{P}^{3}$ or to the blowup of a singular quadric cone in $\mathbb{P}^{3}$ at its vertex. If $Q$ is the class of conic plane sections, the $K=-2 Q$. c) $S$ is isomorphic to a Del Pezzo surface of degree 5 in $\mathbb{P}^{5}$. Its Picard group is cyclic, generated by $K$. The class of hyperplane sections is $-K$.

d) $S$ is isomorphic to a Del Pezzo surface of degree 6 in $\mathbb{P}^{6}$. Its Picard group is again cyclic, generated by $K$, and the class of hyperplane sections is $-K$.

Proof. Let $d:=K^{2}$. By the classification of Del Pezzo surfaces over algebraically closed field (see 11, Theorem 24.4), we have $1 \leq d \leq 9$.

If $d=9$, then $S$ is a Severi-Brauer surface. As $S$ also has a parametrization over $\mathbb{K}$, it is isomorphic to $\mathbb{P}^{2}$ and (a) holds.

If $d=8$, then $S$ is isomorphic to a ruled surface $F_{n}$ over the algebraic closure $\overline{\mathbb{K}}$, where $0 \leq n \leq 2$. The case $n=1$ is not possible, because in this case $S$ would not be minimal; in the two remaining cases we have $-K=2 Q$ for some divisor $Q$, whose associated image is a quadric in $\mathbb{P}^{3}$.

If $d=7$, then $S$ is not minimal.

If $d=6$, then (d) holds.

If $d=5$, then (c) holds.

If $d=4$, then $S$ cannot be both minimal and rational over $\mathbb{K}$, by Theorem 1 in [6]. 
If $d=3,2$, or 1 , then $S$ cannot be both minimal and rational over $\mathbb{K}$, by Theorem 5.7 in 10 .

Theorem 17. Let $S$ be a nonsingular rational surface and let $D$ be a nef divisor such that $\operatorname{keel}(S)=0$ and $D^{2}>0$. Then

$$
\operatorname{pdeg}(D) \leq 6 \operatorname{level}(D)
$$

Proof. By lemmas 13 14 15. we can reduce to the case level $(D)=a$ and $D_{a}=0$ by replacing $D$ by $2 D$ or $3 D$. Then $-K_{a}$ is nef and $\left(-K_{a}\right)^{2}>0$ because $-K_{a}$ is the direct image of $D_{a-1}$. Then $S_{a}$ satisfies the assumptions in Theorem [16] For each of the cases (a), (b), (c), we construct below a parametrizing class $P \in \operatorname{Pic}\left(S_{a}\right)$, such that $P\left(-K_{a}\right) \leq 6$. Let $\phi_{a}: S \rightarrow S_{a}$ be the minimalization map. Then $\phi_{a}^{*}(P)$ is a parametrizing divisor for $S$, and

$$
\phi_{a}^{*}(P) D=P \phi_{a_{*}}(D)=P\left(D_{a}-a K_{a}\right) \leq 6 a .
$$

Case (a): we take $P:=L$. Then $P\left(-K_{a}\right)=3 \leq 6$.

Case (b): we take $P:=Q$. This is a parametrizing class because we can choose a parametrizing system $l$ as the linear system of conic sections through a fixed nonsingular point $p$ defined over $\mathbb{K}$. Such a point exists because $S$ is rational. The associated map is the stereographic projection from $p$, which is birational to $\mathbb{P}^{2}$. In this case, we have $P\left(-K_{a}\right)=2 Q^{2}=4 \leq 6$.

Case (c): we take $P:=-K_{a}$, the class of hyperplane sections. As parametrizing system, we choose the set of all sections with hyperplanes containing the tangent plane through a fixed point $p$ defined over $\mathbb{K}$. The associated map is the projection from the tangent plane, which reduces the dimension by 3 and the degree by 4 , hence it is birational to $\mathbb{P}^{2}$. In this case, we have $P\left(-K_{a}\right)=P^{2}=5 \leq 6$.

Case $(\mathrm{d})$ : again we take $P:=-K_{a}$. As parametrizing system, we choose the set of all sections with hyperplanes containing the tangent plane through a fixed point $p$ and through another fixed point $q$ outside the tangent plane, where both $p$ and $q$ are defined over $\mathbb{K}$. The associated map can be decomposed into the projection from the tangent plane, which is birational onto a quadric in $\mathbb{P}^{3}$, followed by the stereographic projection from the image of $q$. In this case, we have $P\left(-K_{a}\right)=P^{2}=6$.

Theorem 18. Let $S$ be a nonsingular rational surface. Let $P \in \operatorname{Pic}(S)$ be a free class such that $P^{2}=0$ and $P K=-2$ and $\operatorname{dim}(P)=1$. Assume that $S$ is $P$-minimal. Then one of the following cases holds.

a) $S$ is isomorphic to the ruled surface $F_{n}, n \geq 0$. There exists an effective class $C$ such that $C P=1, C^{2}=-n$, and $K=(-n-2) P-2 C$. The classes $C$ and $P$ generate $\operatorname{Pic}(S)$.

b) $S$ is isomorphic to the blowup of a nonsingular quadric at a point of degree 2 (i.e. defined over a quadratic extension of $\mathbb{K}$ ). The Picard group is generated by $P$ and the exceptional class $E$, the class of plane sections is $P+E$, the canonical class is $-2 P-E$, and $P E=2$.

c) $S$ is isomorphic to the blowup of $\mathbb{P}^{2}$ at a point of degree 4. The Picard 
group is generated by the exceptional class $E$ and the class of lines $L$, we have $P=2 L-E$, and the canonical class is $-3 L+E$.

Proof. Because $P$ is free, the associated map $S \rightarrow \mathbb{P}^{1}$ is regular, and $P$ is the class of fibers. The genus of a generic fiber is $\frac{P^{2}+P K}{2}+1=0$, hence the associated map gives $S$ the structur of a conic fibration. Let $d:=K^{2}$. Over the algebraic closure $\overline{\mathbb{K}}$ is a blowup of a ruled surface $F_{n}$ for some $n$, hence $d \leq 8$.

If $d=8$, then $S$ is minimal over $\overline{\mathbb{K}}$, and (a) holds.

If $d=7$, then $S$ is not minimal by Theorem 4.1 in $[5]$.

If $d=6$, then (b) holds by Theorem 4.1 in [5].

If $d=5$, then (c) holds by Theorem 4.1 in [5].

If $d=4$, then $S$ cannot be both minimal and rational over $\mathbb{K}$, by Theorem 2 in 6 .

If $d=3$, then $S$ cannot be both minimal and rational over $\mathbb{K}$, by Corollary 2.6 in $[5]$.

If $d=2$ or 1 , then $S$ cannot be both minimal and rational over $\mathbb{K}$, by Corollary 1.7 in $[5]$.

If $d \leq 0$, then $S$ cannot be both minimal and rational over $\mathbb{K}$, by Theorem 1.6 in 4 .

Theorem 19. Let $S$ be a nonsingular rational surface and let $D$ be a nef divisor such that $D^{2}>0$ and $\operatorname{keel}(S)>0$. Then

$$
\operatorname{pdeg}(D) \leq 4 \operatorname{level}(D)+2 \operatorname{keel}(D) .
$$

Proof. By lemmas 13, 14, 15, and by replacing $D$ by $2 D$, we can reduce to the case level $(D)=a$ and $D_{a}=k P$ for $k=\operatorname{keel}(D)$ and $P$ as in Lemma 14. Then we construct a parametrizing class $Q$ for each of the cases that arise in Theorem 18 If $\phi_{a}: S \rightarrow S_{a}$ be the minimalization map, then $\phi_{a}^{*}(Q)$ is a parametrizing class for $S$; and we will prove the required upper bound for $\phi_{a}^{*}(Q) D$ in each case.

In case (a), we distinguish two subcases. If $S \cong F_{0}$, then we take $Q:=$ $C+P$. The image of the associated map is a ruled quadric in $\mathbb{P}^{3}$. Because a stereographic projection from a point defined over $\mathbb{K}$ is birational onto the plane, the class $Q$ is parametrizing for $S_{a}$, and it follows that $\phi_{a}^{*}(Q)$ is parametrizing for $S$. We compute

$$
\begin{gathered}
\phi_{a}^{*}(Q) D=Q \phi_{a_{*}}(D)=Q\left(D_{a}-a K_{a}\right) \\
=(C+P)(2 a C+2 a P+k P)=4 a+k \leq 4 a+2 k .
\end{gathered}
$$

If $S \cong F_{n}$ and $n>0$, then we take $Q:=C+n P$. Then $\operatorname{dim}(C+n P)=n+1$, and the image $S_{Q} \subset \mathbb{P}^{n+1}$ of the associated map is a cone over a rational normal curve of degree $n$. By repeating projections from nonsingular points defined over $\mathbb{K}$, we obtain a birational map to the plane. Hence $Q$ is parametrizing, and therefore $\phi_{a}^{*}(Q)$ is parametrizing. We have

$$
\phi_{a}^{*}(Q) D=(C+n P)(2 a C+(a n+k+2 a) P)=a n+2 a+k .
$$


Moreover, $D$ is nef, hence

$$
0 \leq \phi_{a}^{*}(D)(C)=2 a+k-a n,
$$

hence $\phi_{a}^{*}(Q) D \leq 4 a+2 k$.

In case (b), we take $Q:=P+E$. The image of the associated map is a quadric in $\mathbb{P}^{3}$, hence $Q$ is parametrizing, as above. In this case, we get

$$
\phi_{a}^{*}(Q) D=(P+E)(k P+2 a P+a E)=4 a+2 k .
$$

In case (c), we take $Q=L$, which is of course parametrizing. In this case, we get

$$
\phi_{a}^{*}(Q) D=L(k P-a K)=L(3 a L+2 k L-(a+k) E)=3 a+2 k \leq 4 a+2 k .
$$

Comprizing Theorems [ 17 19] and Remark 3, we can finally state:

Theorem 20. For any rational surface $S$, the following bounds hold:

$$
3 \operatorname{level}(S)+\operatorname{keel}(S) \leq \operatorname{pdeg}(S) \leq 6 \operatorname{level}(S)+2 \operatorname{keel}(S) .
$$

\section{References}

[1] Mauro C. Beltrametti and Andrew J. Sommese. The adjunction theory of complex projective varieties, volume 16 of de Gruyter Expositions in Mathematics. Walter de Gruyter \& Co., Berlin, 1995.

[2] A. Comesatti. Fondamenti per la geometria sopra le superficie rationali del punto di vista reale. Math. Ann., 73:1-72, 1912.

[3] C. Haase and J. Schicho. Lattice polygons and the number $2 i+7$. Technical Report 2004-04, RICAM, Austrian Academy of Sciences, 2004.

[4] V.A. Iskovskih. Rational surfaces with a pencil of rational curves. Math. USSR Sb., 3:563-587, 1967.

[5] V.A. Iskovskih. Rational surfaces with a pencil of rational curves with positive square of the canonical class. Math. USSR Sb., 12:91-117, 1970.

[6] V.A. Iskovskih. Birational properties of surfaces of degree 4 in $\mathbf{p}^{4}$. Math. USSR Sb., 17:575-577, 1972.

[7] V.A. Iskovskih. Minimal models of rational surfaces over arbitrary fields. Math. USSR Izv., 14:17-39, 1980.

[8] Y. Kawamata, K. Matsuda, and K. Matsuki. Introduction to the minimal model problem. In Algebraic geometry, Sendai, 1985, volume 10 of Adv. Stud. Pure Math., pages 283-360. North-Holland, Amsterdam, 1987. 
[9] Y. Manin. Rational surfaces over perfect fields I. Inst. Hautes Et. Sci. Publ. Math., 30:137-186, 1966.

[10] Y. Manin. Rational surfaces over perfect fields II. Math. USSR Sb., 1:141$168,1967$.

[11] Y. Manin. Cubic Forms. North-Holland, Amsterdam, 1974.

[12] J. Schicho. Rational parametrization of surfaces. J. Symb. Comp., 26(1):130, 1998.

[13] J. Schicho. A degree bound for the parameterization of a rational surface. J. Pure Appl. Alg., 145:91-105, 1999.

[14] J. Schicho. Proper parametrization of real tubular surfaces. J. Symb. Comp., 30:583-593, 2000.

[15] J. Schicho. Simplification of surface parametrizations. In Proc. ISSAC 2002, pages 229-237. ACM Press, 2002. 